\title{
Construction of the Null Scrolls along Lightlike Submanifolds in $\mathbb{R}_{v}^{m+n}$
}

\author{
Gül Tuğ* and F. Nejat Ekmekci \\ (Communicated by Murat Tosun)
}

\begin{abstract}
The theory of null scrolls is still a developing subject. In this paper, the aim is to generalize the null scrolls by developing a method. For this, it is constructed the null scrolls along lightlike submanifolds in $\mathbb{R}_{v}^{m+n}$. Several geometric objects of the defined null scrolls are investigated. The proposed theory is strengthened with examples.
\end{abstract}

Keywords: Lightlike submanifold; null scroll; second fundamental form.

AMS Subject Classification (2010): Primary: 53C50; 58A05 ; Secondary: 53A35 ; $53 C 05$.

\section{Introduction}

It is known that, a ruled surface is generated by a base curve and a non-zero director vector field. Also, the ruled surfaces are classified in five different kinds according to the casual characters of the base curve and the director vector field in a semi- Riemannian manifold. If both of them are null, then the ruled surface is called a null scroll $[8,10]$.

In the semi-Riemannian geometry, the theory of lightlike submanifolds plays an important role. It takes a special place not only in Geometry but also in Physics. In [1], the authors extended the theory of null scrolls by defining $n$-dimensional generalized null scrolls in $\mathbb{R}_{1}^{n}$. However, in the present construction method, it is considered the lightlike submanifolds in order to extend the theory to the higher dimensions and arbitrary indexes. For this, a lightlike normal vector field in the transversal vector bundle is defined by using the bases of $\operatorname{RadTM}, \operatorname{ltr}(T M)$ and $S\left(T M^{\perp}\right)$. It is described by the sum of a timelike unit normal vector field and a spacelike unit normal vector field from the unit spherical bundle. Then the null scroll is defined in two cases depending on the casual characters of the vector fields in the basis of $S\left(T M^{\perp}\right)$ by,

$$
Y_{M}(u, \theta, t)=X(u)+t\left(n^{T}+n^{S}\right)(u), t \in \mathbb{R}
$$

where $X(U)=M$ is the lightlike submanifold, $u=\left(u_{1}, \ldots, u_{m}\right), \theta=\left(\theta_{1}, \ldots, \theta_{n-2}\right)$. Obviously, a more general description of the null scrolls is given in this study. Moreover, the conditions for being flat and the relation between the second fundamental forms of the lightlike submanifold and corresponding null scroll, are given. The theory is strengthened with some examples.

\section{Preliminaries}

In this section, the basic properties of the theory of lightlike submanifolds are mentioned (see [3,4]). Let $\bar{M}$ be a $m+n$ dimensional semi-Riemannian manifold and $\bar{g}$ be the metric with constant index defined on $\bar{M}$. When $M$ is a $m$ dimensional submanifold in $\bar{M}$, for every $p \in M$, consider a subspace

$$
T_{p} M^{\perp}=\left\{v_{p} \in T_{p} \bar{M}: \bar{g}_{p}\left(v_{p}, w_{p}\right)=0, \forall w_{p} \in T_{p} M\right\} .
$$

Then the radical distribution is defined by,

$$
\operatorname{Rad}_{p} M=T_{p} M \cap T_{p} M^{\perp} \neq\{0\}, \forall p \in M .
$$


If the rank of $\operatorname{RadTM}$ is $r>0$, then $M$ is called a $r$-lightlike submanifold.

The complement vector bundle to $\operatorname{Rad} T M$ in $T M$ is $S(T M)$ which is called a screen distribution. Clearly, $S(T M)$, is a non-degenerate for $\bar{g}$. Hence, one can write the following decomposition,

$$
T M=\operatorname{RadTM} \oplus_{\text {ort }} S(T M) .
$$

$\operatorname{Rad} T M=T M \cap T M^{\perp}$ where $T M^{\perp}=\underset{p \in M}{\cup} T_{p} M^{\perp}$.

Theorem 2.1. Let $\left(M, g, S(T M), S\left(T M^{\perp}\right)\right)$ be a r-lightlike submanifold in $\bar{M}$. If $U$ is a coordinate neighborhood of $M$ and $\left\{\xi_{i}\right\}(i=1, \ldots, r)$ is a basis of RadTM. Then, there exist smooth sections $\left\{N_{i}\right\}$ where the following equations hold:

$$
\begin{gathered}
\bar{g}\left(\xi_{i}, N_{i}\right)=\delta_{i j} \\
\bar{g}\left(N_{i}, N_{j}\right)=0, \quad i, j=1, \ldots, r
\end{gathered}
$$

$\operatorname{ltr}(T M)=\operatorname{Span}\left\{N_{i}\right\}$ is called lightlike transversal vector bundle.

The following decomposition is also satisfied;

$$
\left.T \bar{M}\right|_{M}=T M \oplus \operatorname{tr}(T M)
$$

where $\operatorname{tr}(T M)=\operatorname{ltr}(T M) \oplus S\left(T M^{\perp}\right)$. In this case, a quasi-ortonormal basis of $\bar{M}$ along $M$ is

$$
\left\{\xi_{1}, \ldots, \xi_{r}, N_{1}, \ldots, N_{r}, X_{r+1}, \ldots, X_{m}, W_{r+1}, \ldots, W_{n}\right\}
$$

where $\left\{\xi_{1}, \ldots, \xi_{r}\right\}$ is a lightlike basis of $\operatorname{RadTM},\left\{N_{1}, \ldots, N_{r}\right\}$ is a basis of $\operatorname{ltr}(T M),\left\{X_{r+1}, \ldots, X_{m}\right\}$ and $\left\{W_{r+1}, \ldots, W_{n}\right\}$ are the orthogonal basis of $S(T M)$ and $S\left(T M^{\perp}\right)$ respectively.

Then, it can be written

$$
\begin{aligned}
& \bar{\nabla}_{X} Y=\nabla_{X} Y+h(X, Y) \\
& \bar{\nabla}_{X} V=-A_{V} X+\nabla_{X}^{t} V
\end{aligned}
$$

where $\bar{\nabla}$ is the Levi-Civita connection on $\bar{M}, \nabla_{X} Y$ and $\nabla_{X}^{t} V$ are the linear connections on $M$ and $\operatorname{tr}(T M)$ respectively. Note that $\nabla$ is a torsion free induced linear connection. Also, $A_{V} X$ and $h(X, Y)$ are the shape operator and second fundamental form on $M$, respectively.

$L$ and $S$ are the projections of $\operatorname{tr}(T M)$ on $\operatorname{lt}(T M)$ and $S\left(T M^{\perp}\right)$ respectively where $S\left(T M^{\perp}\right) \neq\{0\}$. In this case, $h^{l}$ and $h^{s}$ are the lightlike and screen second fundamental forms respectively defined by

$$
\begin{gathered}
h^{l}(X, Y)=L(h(X, Y)) \text { and } h^{s}(X, Y)=S(h(X, Y)) \\
h(X, Y)=h^{l}(X, Y)+h^{s}(X, Y) .
\end{gathered}
$$

\section{Null Scrolls Along Lightlike Submanifolds}

Let $X(U)=M$ be a $m$ dimensional $r$ - lightlike submanifold in $\mathbb{R}_{v}^{m+n}$ where $n \geq 2$ and $v \leq m .\left\{\xi_{1}, \ldots, \xi_{r}\right\}$ is taken as the basis of $\operatorname{Rad}(T M)$. Then, it is considered that $S\left(T M^{\perp}\right)$ is spanned by the vector fields $\left\{W_{r+1}, \ldots, W_{n}\right\}$. The pseudo orthonormal basis of $S(T M)^{\perp}$ along $M$ is,

$$
\left\{\xi_{1}, \ldots, \xi_{r}, N_{1}, \ldots, N_{r}, W_{r+1}, \ldots W_{n}\right\}
$$

where $\left\{N_{1}, \ldots, N_{r}\right\}$ is a basis of $\operatorname{ltr}(T M)$. Since $S\left(T M^{\perp}\right)$ is a non-degenerate subspace, two cases arise:

Case 1 Let all the vector fields $\left\{W_{r+1}, \ldots, W_{n}\right\}$ be spacelike (timelike). Then, without loss of generality, choose a unit timelike (spacelike) vector field $n^{T}(u)=\frac{a_{1} \xi_{1}(u)+b_{1} N_{1}(u)}{\left\|a_{1} \xi_{1}(u)+b_{1} N_{1}(u)\right\|}$ where $a_{1}, b_{1} \in \mathbb{R}$. On the other hand, take following orthogonal unit spacelike (timelike) vector fields

$$
U_{k-1}(u)=\frac{a_{k} \xi_{k}(u)+b_{k} N_{k}(u)}{\left\|a_{k} \xi_{k}(u)+b_{k} N_{k}(u)\right\|}
$$


where $a_{k}, b_{k} \in \mathbb{R}$ and $k=2, \ldots, r$. Then, one gets a non degenerate subspace $W_{p}=$ $\operatorname{Span}\left\{U_{1}(p), \ldots, U_{r-1}(p), W_{r+1}(p), \ldots, W_{n}(p)\right\}$ in $S(T M)_{p}^{\perp}$ at $p=X(u)$. Therefore, a spacelike (timelike) unit pseudo sphere is,

$$
S_{p}^{n-1}=\left\{v \in W_{p}:\langle v, v\rangle=1\right\} .
$$

Hence, there exists a pseudo spherical bundle $S^{n-1}=\underset{p \in M}{\cup} S_{p}^{n-1}$ over $M$. A unit spacelike vector field $n^{S}(u) \in S^{n-1}$ can be written by means of pseudo spherical parameters $\theta_{j}$ where $j=1, \ldots, n-2$. It is clear that $\left\langle n^{S}, n^{T}\right\rangle=0$. The vector fields $n^{T}(u) \pm n^{S}(u)$ are lightlike. $n^{T}+n^{S}$ is taken as a lightlike transversal normal vector field along $M$.

Definition 3.1. The hypersurface

$$
Y_{M}: S^{n-1} \times \mathbb{R} \longrightarrow \mathbb{R}_{v}^{m+n}
$$

defined by

$$
Y_{M}(u, \theta, t)=X(u)+t\left(n^{T}+n^{S}\right)(u), t \in \mathbb{R}
$$

is called a null scroll along $M$ where $u=\left(u_{1}, \ldots, u_{m}\right), \theta=\left(\theta_{1}, \ldots, \theta_{n-2}\right)$.

Case 2 Now let $\left\{W_{r+1}, \ldots W_{s}\right\}$ be spacelike and $\left\{W_{s+1}, \ldots, W_{n}\right\}$ be timelike vector fields. Then, without loss of generality, choose some orthogonal timelike vector fields as;

$$
\begin{gathered}
n_{1}(u)=\frac{a_{1} \xi_{1}(u)+b_{1} N_{1}(u)}{\left\|a_{1} \xi_{1}(u)+b_{1} N_{1}(u)\right\|} \\
n_{2}(u)=\frac{a_{2} \xi_{2}(u)+b_{2} N_{2}(u)}{\left\|a_{2} \xi_{2}(u)+b_{2} N_{2}(u)\right\|} \\
\vdots \\
n_{\mu}(u)=\frac{a_{\mu} \xi_{\mu}(u)+b_{\mu} N_{\mu}(u)}{\left\|a_{\mu} \xi_{\mu}(u)+b_{\mu} N_{\mu}(u)\right\|}
\end{gathered}
$$

where $a_{1}, \ldots, a_{\mu}, b_{1}, \ldots, b_{\mu} \in \mathbb{U} 211 d, \mu<r$. Similarly, take the following orthogonal spacelike vector fields;

$$
\begin{gathered}
U_{1}(u)=\frac{a_{\mu+1} \xi_{\mu+1}(u)+b_{\mu+1} N_{\mu+1}(u)}{\left\|a_{\mu+1} \xi_{\mu+1}(u)+b_{\mu+1} N_{\mu+1}(u)\right\|} \\
U_{2}(u)=\frac{a_{\mu+2} \xi_{\mu+2}(u)+b_{\mu+2} N_{\mu+2}(u)}{\left\|a_{\mu+2} \xi_{\mu+2}(u)+b_{\mu+2} N_{\mu+2}(u)\right\|} \\
\vdots \\
U_{r-\mu}(u)=\frac{a_{r} \xi_{r}(u)+b_{r} N_{r}(u)}{\left\|a_{r} \xi_{r}(u)+b_{r} N_{r}(u)\right\|}
\end{gathered}
$$

where $a_{\mu+1}, \ldots, a_{r}, b_{\mu+1}, \ldots, b_{r} \in \mathbb{R}$. Then, one gets non degenerate subspaces as follows;

$$
\begin{gathered}
W_{p}^{1}=\operatorname{Span}\left\{n_{1}(p), \ldots, n_{\mu}(p), W_{s+1}(p), \ldots, W_{n}(p)\right\} \\
W_{p}^{2}=\operatorname{Span}\left\{U_{1}(p), \ldots, U_{r-\mu}(p), W_{r+1}(p), \ldots, W_{s}(p)\right\} .
\end{gathered}
$$

Also, the unit pseudo spheres $S_{p}^{\nu}$ and $H_{p}^{\vartheta}$ in $W_{p}^{2}$ and $W_{p}^{1}$ respectively, are defined by

$$
\begin{gathered}
S_{p}^{\varsigma}=\left\{v \in W_{p}^{2}:\langle v, v\rangle=1\right\} \\
H_{p}^{\vartheta}=\left\{v \in W_{p}^{1}:\langle v, v\rangle=-1\right\}
\end{gathered}
$$

where $\varsigma=s-\mu-1, \vartheta=n-s+\mu-1$. Hence, there exist pseudo spherical bundles $S^{\varsigma}=\underset{p \in M}{\cup} S_{p}^{\varsigma}$ and $H^{\vartheta}=\underset{p \in M}{\cup} H_{p}^{\vartheta}$ over $M$. A unit spacelike vector field $n^{S}(u) \in S^{\varsigma}$ and a unit timelike vector field $n^{T}(u) \in H^{\vartheta}$ can be written by means of pseudo spherical parameters $\psi_{j}$ and $\varphi_{l}$, respectively where $j=1, \ldots, \varsigma-1$, $l=1, \ldots, \vartheta-1$. It is clear that, $\left\langle n^{S}, n^{T}\right\rangle=0$. The vector fields $n^{T}(u) \pm n^{S}(u)$ are lightlike. $n^{T}+n^{S}$ is taken as a lightlike transversal normal vector field along $M$. 
Definition 3.2. The hypersurface

$$
Y_{M}: S^{n-1} \times \mathbb{R} \longrightarrow \mathbb{R}_{v}^{m+n}
$$

defined by

$$
Y_{M}(u, \psi, \varphi, t)=X(u)+t\left(n^{T}+n^{S}\right)(u), t \in \mathbb{R}
$$

is called a null scroll along $M$ where $u=\left(u_{1}, \ldots, u_{m}\right), \varphi=\left(\varphi_{1}, \ldots, \varphi_{\vartheta-1}\right), \psi=\left(\psi_{1}, \ldots, \psi_{\varsigma-1}\right)$ and $\vartheta+\varsigma=n$.

Whether or not it belongs to the Case 1 or Case 2, the null scroll along $M$ is denoted by $\tilde{M}$. The tangent space $T_{P} \tilde{M}$ at a point $P \in \tilde{M}$ is spanned by the following vector fields;

$$
\begin{gathered}
Y_{u_{i}}(u, \theta, t)=X_{u_{i}}(u)+t\left(n^{T}+n^{S}\right)_{u_{i}}(u), i=1, \ldots, m \\
Y_{\theta_{j}}(u, \theta, t)=t\left(n_{\theta_{j}}^{S}\right)(u), j=1, \ldots, n-2 \\
Y_{t}(u, \theta, t)=\left(n^{T}+n^{S}\right)(u) .
\end{gathered}
$$

Note that, it is taken $\theta=\left(\theta_{1}, \ldots, \theta_{n-2}\right)=\left(\varphi_{1}, \ldots, \varphi_{\vartheta-1}, \psi_{1}, \ldots, \psi_{\varsigma-1}\right)$ if $\tilde{M}$ belongs to the Case 2. Therefore, $Y_{t}$ is null and $Y_{\theta_{j}}$ are non-null vector fields. $Y_{u_{i}}$ are spacelike, timelike or null vector fields.

Let $Y_{u_{\alpha}}$ be non-null vector fields and $Y_{u_{\beta}}$ be null vector fields where $\alpha=1, \ldots, q$ and $\beta=q+1, \ldots, m$. Take $a_{1}=$ $u_{1}, \ldots, a_{m}=u_{m}, a_{m+1}=\theta_{1}, \ldots, a_{m+n-2}=\theta_{n-2}, a_{m+n-1}=t$ for the easement of the calculations. The induced pseudo Riemannian metric which is the first fundamental form on $\tilde{M}$, is defined by $d s^{2}=\sum_{i, j=1}^{m+n-1} \tilde{g}_{i j} d a_{i} d a_{j}$ where $\tilde{g}_{i j}=\left\langle Y_{a_{i}}, Y_{a_{j}}\right\rangle$. The matrix form of $\tilde{g}_{i j}$ is,

$$
\left[\begin{array}{ccccccccccccc}
\varepsilon_{1} & 0 & \ldots & 0 & 0 & 0 & \ldots & 0 & 0 & \ldots & 0 & 0 & 0 \\
0 & \varepsilon_{2} & \ldots & 0 & 0 & 0 & \ldots & 0 & 0 & \ldots & 0 & 0 & 0 \\
\vdots & \vdots & \ddots & \vdots & \vdots & \vdots & \ddots & \vdots & \vdots & \vdots & \vdots & \vdots & \vdots \\
0 & 0 & \ldots & \varepsilon_{q} & 0 & 0 & \ldots & 0 & 0 & \ldots & 0 & 0 & 0 \\
0 & 0 & \ldots & 0 & 0 & \lambda_{q+1}^{q+2} & \ldots & \lambda_{q+1}^{m} & 0 & \ldots & 0 & 0 & \lambda_{m+n-1}^{q+1} \\
0 & 0 & \ldots & 0 & \lambda_{q+2}^{q+1} & 0 & \ldots & \lambda_{q+2}^{m} & 0 & \ldots & 0 & 0 & \lambda_{m+n-1}^{q+2} \\
\vdots & \vdots & & \vdots & \vdots & & \ddots & & \vdots & & \vdots & \vdots & \vdots \\
0 & 0 & & 0 & \lambda_{m}^{q+1} & \lambda_{m}^{q+2} & \ldots & 0 & 0 & \ldots & 0 & 0 & \lambda_{m+n-1}^{m} \\
0 & 0 & \ldots & 0 & 0 & 0 & \ldots & 0 & \varepsilon_{m+1} & \ldots & 0 & 0 & 0 \\
0 & 0 & & 0 & 0 & 0 & & 0 & 0 & \varepsilon_{m+2} & 0 & 0 & 0 \\
\vdots & \vdots & & \vdots & \vdots & \vdots & & \vdots & \vdots & & \ddots & \vdots & \vdots \\
0 & 0 & \ldots & 0 & 0 & 0 & \ldots & 0 & 0 & \ldots & 0 & \varepsilon_{m+n-2} & 0 \\
0 & 0 & & 0 & \lambda_{m+n-1}^{q+1} & \lambda_{m+n-1}^{q+2} & \ldots & \lambda_{m+n-1}^{m} & 0 & \ldots & 0 & 0 & 0
\end{array}\right]
$$

where $\varepsilon_{k}= \pm 1$ and $\lambda_{i}^{j}=0$ or 1 depending on the null vector fields.

Now consider a normal vector field $V \in N_{P} \tilde{M}$ along $\tilde{M}$. Then the second fundamental form of $\tilde{M}$ is,

$$
\tilde{h}_{i j}=\left\langle-\tilde{\nabla}_{Y_{a_{i}}} V, Y_{a_{j}}\right\rangle, i, j=1, \ldots, m+n-1
$$

where $\tilde{\nabla}$ is the induced connection on $\tilde{M}$.

Theorem 3.1. A null scroll along a r-lightlike submanifold is flat.

Theorem 3.2. A null scroll along a $r$-lightlike submanifold is Ricci-flat.

Proposition 3.1. Let $\tilde{M}$ be a null scroll along a $r$-lightlike submanifold $M$. Then, the Weingarten formulas on $\tilde{M}$ are,

$$
\tilde{\nabla}_{Y_{a_{i}}} V=-\sum_{j=1}^{m+n-1} \tilde{h}_{i}^{j} Y_{a_{j}}, i=1, \ldots, m+n-1
$$

where $\tilde{h}_{i}^{j}=\tilde{h}_{i k} \tilde{g}^{k j}$ and $\tilde{g}^{k j}=\left(\tilde{g}_{k j}\right)^{-1}$. 
Theorem 3.3. For a null scroll along a $r$-lightlike submanifold, the following equations hold;

$$
\begin{array}{rlc}
\tilde{h}_{\alpha}^{\sigma} & =\tilde{h}_{\alpha}^{\alpha} \tilde{h}_{\sigma}^{\sigma} \varepsilon_{\alpha} \varepsilon_{\sigma} \\
\tilde{h}_{i}^{j} & = & \tilde{h}_{i}^{i} \tilde{h}_{j}^{j} \varepsilon_{i} \varepsilon_{j} \\
\tilde{h}_{j}^{j} \tilde{h}_{\alpha}^{\alpha} & = & 0
\end{array}
$$

where $i, j=m+1, \ldots, m+n-2, \alpha, \sigma=1, \ldots, q$.

Theorem 3.4. Let $h_{i j}^{l}$ and $h_{i j}^{s}$ be the lightlike and screen second fundamental forms of a $r$ - lightlike submanifold M respectively. Then, they are related to the second fundamental form of the corresponding null scroll by following equation:

$$
\begin{aligned}
\tilde{h}_{i j}= & \sum_{l=1}^{r} c_{l}\left[h_{i j}^{l}+t \sum_{k=1}^{m-r}\left(\eta_{j k} h_{i k}^{l}+\eta_{i k} h_{k j}^{l}+t \eta_{i k} \eta_{j k} h_{k k}^{l}\right)\right] \\
& +\sum_{s=r+1}^{n} d_{s}\left[h_{i j}^{s}+t \sum_{k=1}^{m-r}\left(\eta_{j k} h_{i k}^{s}+\eta_{i k} h_{k j}^{s}+t \eta_{i k} \eta_{j k} h_{k k}^{s}\right)\right]
\end{aligned}
$$

where $i, j=1, \ldots, m$ and $c_{l}, d_{s}, \eta_{i k} \in \mathbb{R}$.

Corollary 3.1. If all the null vector fields in the pseudo-frame of the tangent space at every point on the null scroll $\tilde{M}$ are orthogonal, then $\tilde{M}$ is totally umbilical.

Theorem 3.5. A null scroll along a r-lightlike submanifold is totally geodesic iff;

$$
\begin{gathered}
\tilde{h}_{i}^{\alpha}=0 \\
\tilde{h}_{i}^{j}=0 \\
\sum_{k=q+1}^{m} \tilde{h}_{i}^{k} \lambda_{\beta}^{k}=0 \\
\sum_{k=q+1}^{m} \tilde{h}_{i}^{k} \lambda_{(m+n-1)}^{k}=0
\end{gathered}
$$

where $i=1, \ldots, m+n-1, j=m+1, \ldots, m+n-1, \alpha=1, \ldots, q, \beta=q+1, \ldots, m$.

Corollary 3.2. Let $M$ be a totally geodesic $r$-lightlike submanifold. Then, the vector fields $Y_{u_{i}}(i=1, \ldots, m)$ are asymptotic directions of the corresponding null scroll $\tilde{M}$.

\section{Examples}

Example 4.1. Take the following null curve as the lightlike submanifold $M$,

$$
\alpha(s)=\left(\frac{1}{2} \sinh (2 s), \frac{1}{2} \cosh (2 s), s\right) .
$$

The Cartan frame along $\alpha$ is,

$$
\begin{gathered}
L=(\cosh (2 s), \sinh (2 s), 1) \\
N=\left(\frac{1}{2} \cosh (2 s), \frac{1}{2} \sinh (2 s),-\frac{1}{2}\right) \\
W=(\sinh (2 s), \cosh (2 s), 0) .
\end{gathered}
$$

Choose $n^{T}=\frac{L+N}{\|L+N\|}=\left(\frac{3}{2 \sqrt{2}} \cosh (2 s), \frac{3}{2 \sqrt{2}} \sinh (2 s), \frac{1}{2 \sqrt{2}}\right)$ and $n^{S}=W$. Therefore, the vector field

$$
n^{T}+W=\left(\frac{3}{2 \sqrt{2}} \cosh (2 s)+\sinh (2 s), \frac{3}{2 \sqrt{2}} \sinh (2 s)+\cosh (2 s), \frac{1}{2 \sqrt{2}}\right)
$$

is a lightlike transversal normal vector field along $\alpha(s)$. Hence, the null scroll along $\alpha(s)$ is;

$$
Y(s, t)=\alpha(s)+t\left(n^{T}+W\right) .
$$




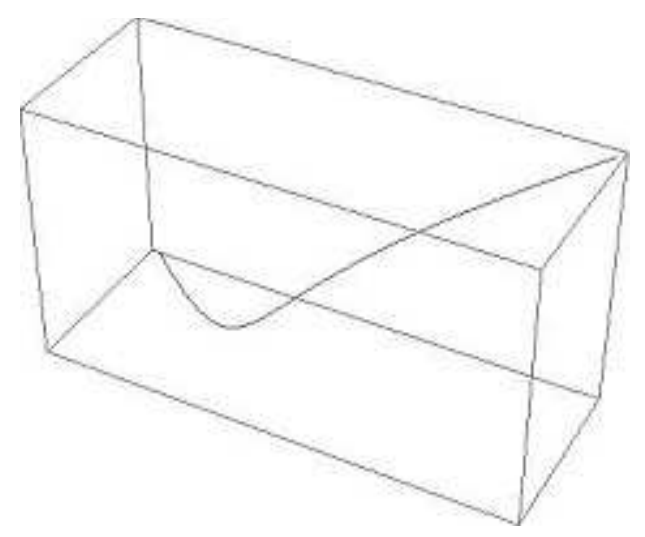

Figure 1. Curve $\alpha(s)$

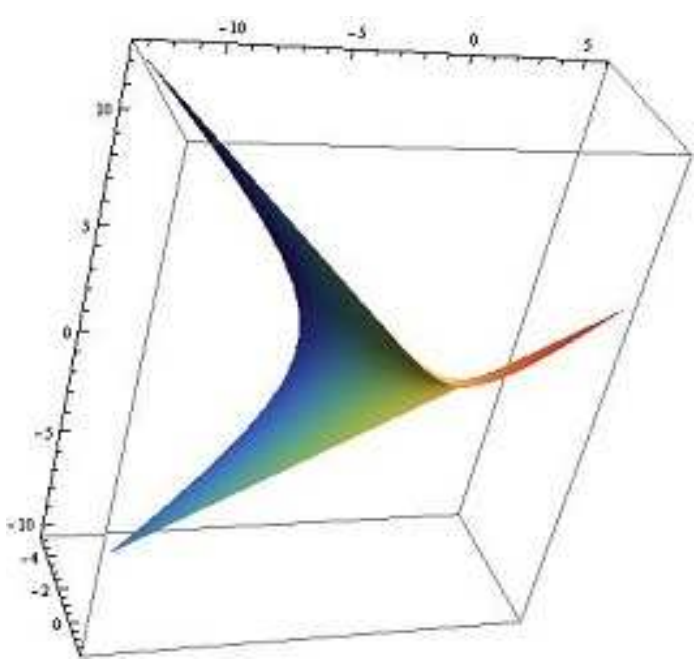

Figure 2. Null scroll $Y(s, t)$

Example 4.2. In $\mathbb{R}_{2}^{5}$, take a lightlike submanifold $M=X\left(x_{1}, x_{2}\right)$ as,

$$
x_{3}=\cos x_{1}, x_{4}=\sin x_{1}, x_{5}=x_{2} .
$$

Then $\operatorname{RadTM}=T M=\operatorname{Span}\left\{\xi_{1}, \xi_{2}\right\}$ and $T M^{\perp}=\operatorname{Span}\left\{\xi_{1}, W_{1}, W_{2}\right\}$ where,

$$
\begin{gathered}
\xi_{1}=\partial_{2}+\partial_{5} \\
\xi_{2}=\partial_{1}-\sin x_{1} \partial_{3}+\cos x_{1} \partial_{4} \\
W_{1}=-\sin x_{1} \partial_{1}+\partial_{3} \\
W_{2}=\cos x_{1} \partial_{1}+\partial_{4} .
\end{gathered}
$$

Therefore, $\operatorname{lt} T M=\operatorname{Span}\left\{N_{1}, N_{2}\right\}$ where

$$
N_{1}=\frac{1}{2}\left[-\partial_{2}+\partial_{5}\right] \text { and } N_{2}=\frac{1}{2}\left[-\partial_{1}-\sin x_{1} \partial_{3}+\cos x_{1} \partial_{4}\right] .
$$

Since $\left\{W_{1}, W_{2}\right\}$ are spacelike, it is the Case 1 . Now, choose the unit timelike vector field

$$
n^{T}=\frac{-\xi_{1}+N_{1}}{\left\|-\xi_{1}+N_{1}\right\|}=-\frac{3}{2 \sqrt{2}} \partial_{2}-\frac{1}{2 \sqrt{2}} \partial_{5}
$$

Then, the vector field

$$
U=\frac{\xi_{2}+N_{2}}{\left\|\xi_{2}+N_{2}\right\|}=\frac{1}{2 \sqrt{2}} \partial_{1}-\frac{3}{2 \sqrt{2}} \sin x_{1} \partial_{3}+\frac{3}{2 \sqrt{2}} \cos x_{1} \partial_{4}
$$


is spacelike and the subspace $W_{p}=\operatorname{Span}\left\{U, W_{1}, W_{2}\right\}_{p}$ where $p=X(u)$. One can take a spacelike unit vector field $n^{S} \in S^{n-1}$ as

$$
n^{S}=\cos \theta_{1} U+\sin \theta_{1} \cos \theta_{2} W_{1}+\sin \theta_{1} \sin \theta_{2} W_{2} .
$$

Then $n^{T}+n^{S}$ is a lightlike transversal normal vector field and

$$
Y\left(x_{1}, x_{2}, \theta_{1}, \theta_{2}, t\right)=X\left(x_{1}, x_{2}\right)+t\left(n^{T}+n^{S}\right)\left(x_{1}, x_{2}\right)
$$

is a null scroll along $M$.

Example 4.3. In $\mathbb{R}_{4}^{14}$, take a lightlike submanifold $M=X(\mathrm{x})$ as,

$$
\begin{gathered}
x_{1}=x_{14} \\
x_{2}=-x_{13} \\
x_{3}=x_{12} \\
x_{7}=\sqrt{1-\left(x_{8}\right)^{2}} .
\end{gathered}
$$

where

$$
\mathbf{x}=\left(x_{1}, x_{2}, x_{3}, x_{4}, x_{5}, x_{6}, x_{8}, x_{9}, x_{10}, x_{11}\right) .
$$

Then, $T M=\operatorname{Span}\left\{Z_{1}, Z_{2}, Z_{3}, Z_{4}, Z_{5}, Z_{6}, Z_{7}, Z_{8}, Z_{9}, Z_{10}\right\}$ where

$$
\begin{gathered}
Z_{1}=\partial_{1}+\partial_{14} \\
Z_{2}=\partial_{2}-\partial_{13} \\
Z_{3}=\partial_{3}+\partial_{12} \\
Z_{4}=\partial_{4} \\
Z_{5}=\partial_{5} \\
Z_{6}=\partial_{6} \\
Z_{7}=-x_{8} \partial_{7}+x_{7} \partial_{8} \\
Z_{8}=\partial_{9} \\
Z_{9}=\partial_{10} \\
Z_{10}=\partial_{11}
\end{gathered}
$$

and

$$
\begin{gathered}
\operatorname{Rad} T M=\operatorname{Span}\left\{Z_{1}, Z_{2}, Z_{3}\right\} \\
S\left(T M^{\perp}\right)=\operatorname{Span}\left\{W=x_{7} \partial_{7}+x_{8} \partial_{8}\right\} \\
\operatorname{ltr}(T M)=\operatorname{Span}\left\{N_{1}=\frac{1}{2}\left[-\partial_{1}+\partial_{14}\right], N_{2}=\frac{1}{2}\left[-\partial_{2}-\partial_{13}\right], N_{3}=\frac{1}{2}\left[-\partial_{3}+\partial_{12}\right]\right\} .
\end{gathered}
$$

Now, choose the timelike unit normal and spacelike vector fields as,

$$
\begin{aligned}
n^{T} & =\frac{Z_{1}-N_{1}}{\left\|Z_{1}-N_{1}\right\|}=\frac{3}{2 \sqrt{2}} \partial_{1}+\frac{1}{2 \sqrt{2}} \partial_{14} \\
U_{1} & =\frac{Z_{2}+N_{2}}{\left\|Z_{2}+N_{2}\right\|}=\frac{1}{2 \sqrt{2}} \partial_{2}-\frac{3}{2 \sqrt{2}} \partial_{13} \\
U_{2} & =\frac{Z_{3}+N_{3}}{\left\|Z_{3}+N_{3}\right\|}=\frac{1}{2 \sqrt{2}} \partial_{3}+\frac{3}{2 \sqrt{2}} \partial_{12}
\end{aligned}
$$

Not that, $\left\langle U_{1}, U_{2}\right\rangle=0,\left\langle U_{1}, W\right\rangle=\left\langle U_{2}, W\right\rangle=0$. Therefore, it can be written that

$$
n^{S}=\cos \theta_{1} U_{1}+\sin \theta_{1} \cos \theta_{2} U_{2}+\sin \theta_{1} \sin \theta_{2} W .
$$

Hence,

$$
Y\left(\mathbf{x}, \theta_{1}, \theta_{2}, t\right)=\left[X+t\left(n^{T}+n^{S}\right)\right](\mathbf{x})
$$

is a null scroll along $M$. 


\section{Conclusion}

In this study, the theory of null scrolls is generalized with the proposed method. The method provides defining null scrolls in the higher dimensions and arbitrary indexes. Also, with the help of the base lightlike submanifold, one can determine the intrinsic objects of the null scrolls. For example, a relation between the second fundamental forms of the base lightike submanifold and the null scrolls, is given. Moreover, some geometric properties of the null scrolls, such as the flatness, being totally umbilic and totally geodesic, are investigated. Lastly, the constructed theory is supported by several examples.

\section{References}

[1] Balgetir, H. and Ergüt, M., Generalized null scrolls in the $n$-dimensional Lorentzian space. Act. Math. Vietn., 2 (2004), 205-216.

[2] Balgetir, H. and Ergüt, M., (n - 1)-dimensional generalized null scrolls in $R_{1}^{n}$. Acta Math. Aca. Pae. Nyir., 19 (2003), $227-231$.

[3] Duggal, K. and Bejancu, A., Lightlike Submanifolds Of Semi-Riemannian Manifolds and Applications. Math. and Its App., 1996.

[4] Duggal, K. and Şahin, B., Differential Geometry Of Lightlike Submanifolds. Birk. Ver. AG., 2010.

[5] Izumiya, S. and Sato, T., Lightlike hypersurfaces along spacelike submanifolds in Minowski space-time. Journal of Geometry and Physics, 71 (2013), 3052.

[6] Graves, L. K., Codimension one isometric immersions between Lorentz spaces. Trans. of The Amer. Math. Soc., 252 (1979), 367392.

[7] Güner, G. and Ekmekci, N., On the special curves in Minkowski 4 spacetime. Math. Aeterna, 4 (2014), 863-867.

[8] Kim, Y. H. and Yoon, D. W., Classification of ruled surfaces in Minkowski 3-spaces. Journal of Geometry and Physics, 49 (2004), 89-100.

[9] O’Neill, B., Semi Riemannian Geometry With Applications To Relativity. Academic Press, 1983.

[10] Pak, J. S. and Yoon, D. W., On null scrolls satisfying the condition $\Delta H=A H$. Comm. Korean Math. Soc. 3 (2000), 533-540.

GÜL TUĞ

ADDRESS: Karadeniz Technical University, Department of Mathematics, Trabzon, Turkey

E-MAIL: gguner@ktu.edu.tr

F. NEJAT EKMEKCI

ADDRESS: Ankara University, Department of Mathematics, Ankara, Turkey

E-MAIL: ekmekci@science.ankara.edu.tr 